\title{
Measuring the Magnetic Field Amplitude of rf Radiation by the Quasistatic Magnetic Field Effect in Organic Light-Emitting Diodes
}

\author{
Tobias Grünbaum $\odot,{ }^{1}$ Sebastian Bange, ${ }^{1}$ Wei Jiang $\odot,{ }^{2}$ Anna E. Leung $\odot,{ }^{3}$ Tamim A. Darwish $\odot,{ }^{3}$ \\ Paul L. Burn, ${ }^{2}$ and John M. Lupton ${ }^{1, *}$ \\ ${ }^{1}$ Institut für Experimentelle und Angewandte Physik, Universität Regensburg, Universitätsstraße 31, 93053 \\ Regensburg, Germany \\ ${ }^{2}$ Centre for Organic Photonics \& Electronics, School of Chemistry and Molecular Biosciences, The University of \\ Queensland, Brisbane, Queensland 4072, Australia \\ ${ }^{3}$ National Deuteration Facility, Australian Nuclear Science and Technology Organization (ANSTO), Locked Bag \\ 2001, Kirrawee DC, NSW 2232, Australia
}

(Received 8 January 2021; revised 19 April 2021; accepted 30 April 2021; published 1 June 2021)

\begin{abstract}
Electron paramagnetic resonance (EPR) is a versatile tool to probe spin physics in organic semiconductor materials. A common method used to detect the spin- $1 / 2$ paramagnetic resonance in organic light-emitting diodes (OLEDs) is to measure the device resistance under EPR conditions, i.e., to record electrically detected magnetic resonance (EDMR). Here, we present ultralow-frequency EDMR experiments on OLEDs that exhibit a qualitatively new line shape because of a quasistatic magnetic field effect: the modulation of the static ultrasmall field-effect magnetoresistance arising from the magnetic field amplitude $B_{1}$ of the radio frequency (rf) radiation. The disappearance of spin- $1 / 2$ Zeeman resonances of individual charge carriers in the OLED, i.e., the resonances at magnetic fields where the Zeeman splitting matches the photon energy of the incident $r$ radiation, coincides with the emergence of the quasistatic effect. We discuss the origin of this quasistatic magnetic field effect, its characteristic line shape in terms of the magnetic field dependence, the influence of experimental parameters, and the application potential with regards to EDMR experiments. The EDMR line shape can be inferred numerically from the magnetoresistance measurements. This approach enables a unique means of determining the drive-field strength $B_{1}$ in EDMR under driving conditions where alternative methods employing an analysis of the Zeeman resonance — such as power broadening and Rabi flopping — are not applicable.
\end{abstract}

DOI: 10.1103/PhysRevApplied.15.064001

\section{INTRODUCTION AND MOTIVATION}

Organic light-emitting diodes (OLEDs) in a magnetic field display the most elementary quantum system - the two-level system - due to the Zeeman splitting of the energy of individual charge-carrier spins. This characteristic can be directly visualized by exciting resonant transitions between the two spin states, up or down, and monitoring the Rabi oscillations of the state occupation by pulsed magnetic-resonance experiments [1,2]. Even static magnetic fields $B_{0}$ as small as $120 \mu \mathrm{T}$, barely three times the geomagnetic field strength, can lead to distinctly resolved resonance peaks at room temperature under radio frequency (rf) excitation, although the energy of the Zeeman splitting is more than seven orders of magnitude smaller than the thermal energy [3]. The reason that such a high resolution can be achieved lies in the fact

*john.lupton@physik.uni-regensburg.de that the resonance occurs in nonequilibrium states, electrically injected electron-hole pairs bound by the Coulomb interaction, which can exist either in a singlet or a triplet spin configuration. These carrier pairs can either dissociate back to free charges or combine to form molecular excitons, singlets or triplets, which are split in energy by a strong exchange interaction. In the OLEDs used here, it is the singlets that are responsible for the electroluminescence (EL). Application of a magnetic field changes the singlet:triplet ratio of the carrier pairs and consequently affects the emissive exciton yield as well as the density of free charge carriers due to the different dissociation and recombination rates for singlet and triplet spin pairs. This reasoning is known as the "radical-pair model" of magnetoresistance and magnetoEL in organic semiconductors [4-6], and was originally derived to rationalize spin-pair processes responsible for magnetic field effects in chemical reactions [7-9].

Within the radical-pair model, resonant excitation of Zeeman-split energy levels of individual spins induces 
observable changes in device resistance and EL. A change of orientation of a single spin will modify the spin-permutation symmetry of the associated carrier pair, thereby influencing the yields of free charge carriers and excitons $[1,10]$. For fluorescent, i.e., singletemitting materials, the resolution of the resonance lines is not limited by spin-orbit coupling, which is exceedingly weak, but rather by the inhomogeneity of the coupling of charge-carrier spins to the surrounding nuclei, the hyperfine fields. As a consequence, for excitation frequencies $f>10 \mathrm{MHz}$, spectra consisting of two Gaussian lines are observed, reporting on the hyperfine field distributions experienced by electron and hole spins [11]. Previously, we showed that a low-frequency limit for resolving such Zeeman resonances in OLEDs exists [3,12], and is around $3 \mathrm{MHz}$ for devices based on the perdeuterated version of poly[2-methoxy-5-(2'-ethylhexyloxy)-1,4phenylenevinylene] (d-MEHPPV). At even lower values of the excitation frequency, the shape of the EDMR spectra changes, exhibiting a single peak centered around $B_{0}=0 \mathrm{mT}$ accompanied by an inversion of the signal for nonzero $B_{0}$. In Ref. [3], we noted the similarity of these signals to the curvature of the static magnetic field effect, i.e., the magnetoresistance [13]. We note that this near-zero-frequency regime of oscillating magnetic field effects was previously predicted theoretically in radicalpair effects in reaction-yield-detected magnetic resonance (RYDMR) [14] and observed experimentally [15,16]. In these experiments, the magnetic field was kept constant and the excitation frequency was swept. Later experiments in the limit of vanishing $B_{0}$, where the magnetic field in RYDMR was swept under constant frequency, show results more similar to those of our present investigations [17-19]. In this earlier work, it was shown that for $f \rightarrow 0$ the signals obtained arise as a consequence of the excitation field $\mathbf{B}_{1}$ acting as a significant quasistatic contribution to the total magnetic field $\mathbf{B}_{\text {tot }}$ [18].

By exploring a significantly larger part of the full parameter space of the EDMR experiments at lower frequencies than in Ref. [3], including parallel driving conditions where $\mathbf{B}_{1} \| \mathbf{B}_{0}$, we are now able to provide a conclusive reasoning for the observed zero-field phenomena in OLED EDMR. Following an intuitive picture of the quasistatic magnetic field effect, a numerical procedure can be performed to compute the line shape and signal amplitude expected in a low-frequency magneticresonance experiment without the need of any quantitative microscopic assumptions or parameters. The computation can be applied to determine the excitation-field strength $B_{1}$ for very-low-frequency rf excitation of the spin transitions, where the model and experiment show very good agreement. This result is an important achievement since the few other methods available to assess the strength of $\mathbf{B}_{1}$ - analysis of the Lorentzian power broadening of the EDMR spectrum $[12,20]$ or of the Rabi-flopping frequency
$[1,21]$ - crucially rely on the Zeeman resonance, which is either not the dominant feature or is even entirely absent in the parameter regime investigated here.

\section{EXPERIMENTAL SECTION}

\section{A. Ultralow-frequency EDMR of d-MEHPPV OLEDs}

The OLEDs studied are comprised of a novel perdeuterated version of the commonly used emitter material MEHPPV [22]. Devices are fabricated as described in Ref. [3]. The setup for performing the EDMR experiments, depicted in Fig. 1(a), consists of a 3D set of Helmholtz coils (Serviciencia S.L.U., model Ferronato BH300-3-A) to produce homogeneous magnetic fields of up to $B_{0}=2 \mathrm{mT}$ and a stripline for $\mathrm{rf}$ excitation, generating the oscillating field $\mathbf{B}_{1}$. The $3 \mathrm{D}$ coil arrangement enables the angle $\alpha$ between $\mathbf{B}_{1}$ and $\mathbf{B}_{0}$ to be freely adjusted. Furthermore, the effect of the Earth's magnetic field is compensated for by the Helmholtz coil set. The OLEDs are operated under constant-current conditions at $I=100 \mu$ A using a Keithley 238 source-measure unit, and the voltage is measured simultaneously. In this configuration, the measurements are predominantly sensitive to spin-dependent recombination as opposed to spin-dependent transport [23]. Lock-in detection with $100 \%$ square-wave modulation of the $\mathrm{rf}$ excitation at a frequency of $232 \mathrm{~Hz}$ is used to detect only signals induced by the rf excitation $[12,24]$. To record an EDMR spectrum, the external static magnetic field $B_{0}$ is swept from -2 to $2 \mathrm{mT}$ while keeping the excitation frequency $f$ constant. All measurements are performed at room temperature.

In order to illustrate the transition from conventional Zeeman resonances to the quasistatic magnetic field effect, we begin by discussing the frequency dependence of the EDMR signal of the d-MEHPPV-containing OLEDs. EDMR spectra are recorded for frequencies from 0.5 to $3.5 \mathrm{MHz}$ at an excitation power of $1.26 \mathrm{~W}$, which corresponds to an excitation-field strength $B_{1}=49 \mu \mathrm{T}$. This estimate of $B_{1}$ stems from a magnetostatic analysis of the stripline geometry detailed in Sec. I of the Supplemental Material [25] and is further corroborated by the analysis of the power broadening of the Zeeman resonance at higher frequencies $f$, as outlined previously $[12,20]$. Figure 1(b) shows the result for perpendicular excitation, $\mathbf{B}_{1} \perp \mathbf{B}_{0}$ : while the Zeeman resonances at $B_{0} \approx \pm 125 \mu \mathrm{T}$ can still be clearly resolved at $f=3.5 \mathrm{MHz}$, only one central peak at $B_{0}=0 \mathrm{mT}$ is detected for $f=2 \mathrm{MHz}$. For excitation frequencies below $f=1.5 \mathrm{MHz}$, the signal partially inverts, in agreement with our earlier observations [3]. The experiment is repeated for a configuration where the excitation field is parallel to the static external field, $\mathbf{B}_{1} \| \mathbf{B}_{0}$. As displayed in Fig. 1(c), the signals show a pronounced three-peak structure, with even stronger signal inversion than under the perpendicular orientation of the drive field. It is not possible to resolve distinct Zeeman resonances in 
(a)

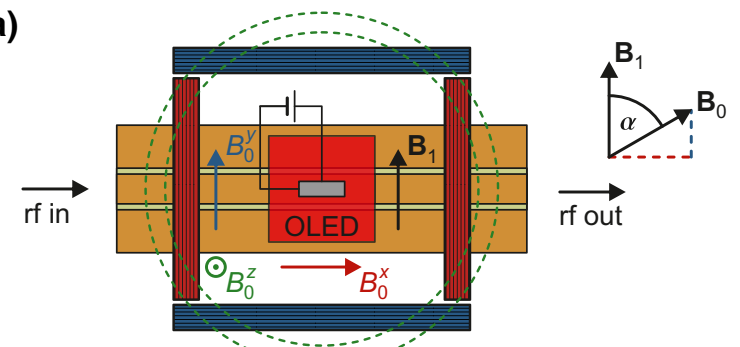

(b)

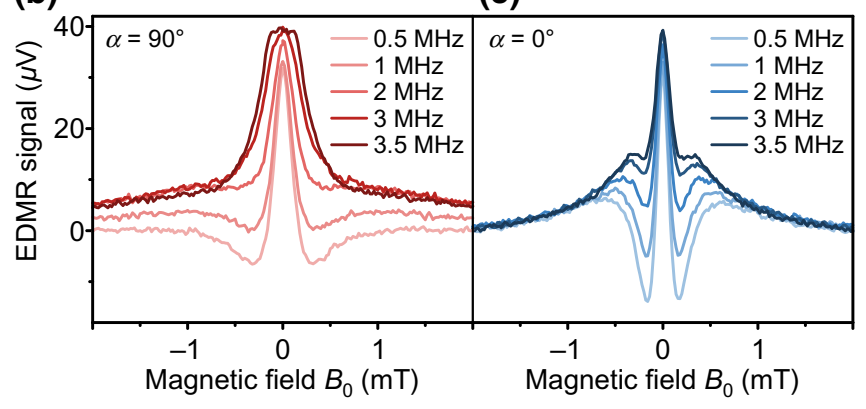

FIG. 1. (a) Schematic of the experimental setup including the stripline and the OLED. The Helmholtz coils generating magnetic fields in the $x, y$, and $z$ directions are indicated in red, blue, and green, respectively. (b) Frequency dependence of the OLED EDMR signal under perpendicular excitation $(\alpha=$ $90^{\circ}$, red lines). Zeeman resonance peaks are distinguished for $f=3.5 \mathrm{MHz}$. At lower frequencies, partial inversion of the signal occurs. (c) The signal inversion is more pronounced under parallel excitation $\left(\alpha=0^{\circ}\right.$, blue lines). All measurements are performed at an excitation power of $1.26 \mathrm{~W}$ corresponding to $B_{1}=49 \mu \mathrm{T}$.

this case, which is to be expected since efficient excitation of the $\Delta m=1$ resonance is not possible with photons polarized parallel to $\mathbf{B}_{0}$ (so-called $\pi$-photons) as long as $\mathbf{B}_{0}$ is the dominating magnetic field contribution $[3,19,28]$. For both excitation geometries, the EDMR spectra bear striking similarity to RYDMR spectra with a low-field structure attributed to the oscillating magnetic field effect [14,17-19].

\section{B. Quasistatic magnetic field effect}

With a wealth of unusual features contained in the low-frequency EDMR spectra, a rationale is sought for the nonmonotonic line shape and the disappearance of the Zeeman resonances at low frequencies. We note that for our experiments, the period of the $\mathrm{rf}$ driving field $\mathbf{B}_{1}(T=2 \mu$ s for $f=0.5 \mathrm{MHz})$ is longer than the typical room-temperature spin-coherence times of $T_{2} \sim 300 \mathrm{~ns}$ in organic semiconductors $[22,29]$. As a consequence, the charge-carrier spins of most carrier pairs in the OLED exhibit phase coherence for only a small fraction of the rf excitation period, and therefore tend to experience the $\mathbf{B}_{1}$ field rather as a static field than as an oscillating, resonance-inducing one $[18,19]$. This qualitative consideration can serve as an explanation for the gradual disappearance of the Zeeman resonances with decreasing excitation frequency: fewer and fewer pairs in the OLED charge-carrier ensemble retain their coherence for timescales much longer than the rf period. Together with the decreasing number of charge-carrier pairs that can fulfil the resonance condition in the presence of isotropic hyperfine fields, this reduction in the ensemble of spins undergoing coherent precession in the resonant driving field effectively limits the observable EDMR signal arising due to changes in spin-permutation symmetry under resonant excitation.

It is well known that radical-pair-based systems not only exhibit resonant magnetic field effects but also respond in a characteristic manner to moderate static external fields $[6-9,13,30,31]$. This behavior, the decrease of the OLED resistance with increasing magnetic field on the scale of several tens of millitesla, along with a nonmonotonic low-field structure for $B_{0}<1 \mathrm{mT}$, is also found for the d-MEHPPV OLEDs investigated here. In Fig. 2(a), the relative resistance change due to a magnetic field, i.e., the magnetoresistance, is shown. Close inspection of the field range of the EDMR experiments in Fig. 1 reveals nonmonotonic behavior, which arises as a consequence of electronic and electron-nuclear spin-spin interactions $[13,32]$. The shape of this feature is therefore dependent on microscopic material properties, such as the hyperfine interactions, the exchange and spin-dipolar interactions within the carrier pairs, and the recombination and dissociation rates of singlet and triplet pairs. In other words, a wealth of microscopic information is contained in the shape of this ultrasmall magnetic field effect.

A crucial consequence of the quasistatic character of the excitation field with regards to the timescale of $T_{2}$ is that the total external magnetic field at each point in time is determined by the vector sum of the static and oscillating contributions as depicted in Fig. 2(b) $[18,19]$ : $\mathbf{B}_{\text {tot }}(t)=$ $\mathbf{B}_{0}+\mathbf{B}_{1}(t)=\mathbf{B}_{0}+\mathbf{B}_{1} \cos (2 \pi f t)$. Spin pairs that retain their coherence in precession with respect to the driving field can exist at different time intervals during the rf period and therefore experience different effective values of the total magnetic field. Each pair then contributes to the magnetoresistance signal corresponding to the value of $\mathbf{B}_{\text {tot }}$ during its coherence time. The lock-in signal, which reports on the effect of the amplitude-modulated rf excitation on resistivity, is then generated as the sum of the contributions of all phases of the rf period. At a given $\mathbf{B}_{0}$, this sum is determined by $\left\langle R\left(B_{\text {tot }}\right)\right\rangle-R\left(B_{0}\right)$, where $\left\langle R\left(B_{\text {tot }}\right)\right\rangle$ is the device resistance averaged over all phases of the rf period. For collinear orientation of $\mathbf{B}_{1}$ and $\mathbf{B}_{0}$, the vectorial character of the calculation of $\mathbf{B}_{\text {tot }}$ disappears, and the signal essentially measures the curvature of the magnetoresistance profile. At the inflection points of $R\left(B_{0}\right)$, $\left\langle R\left(B_{\mathrm{tot}}\right)\right\rangle=R\left(\left\langle B_{\mathrm{tot}}\right\rangle\right)=R\left(B_{0}\right)$ and the quasistatic-effect 
(a)

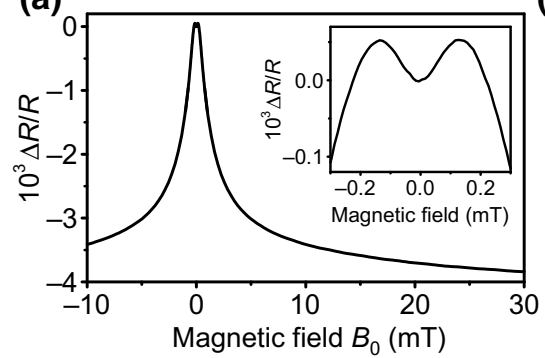

(b)

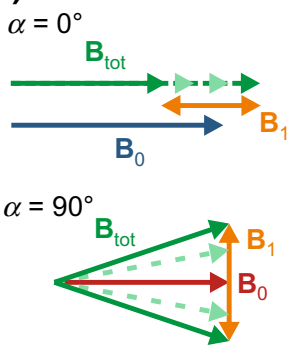

(c)

(d)
We now compare the predictions of this simple model to EDMR spectra taken at a very low frequency of $f=0.5 \mathrm{MHz}$ and a power of $P=1.26 \mathrm{~W}$. The device current is kept constant at $I=100 \mu \mathrm{A}$. Given our earlier discussion, such spectra should, of course, not technically be referred to as "resonance" spectra, even though the EDMR measurement procedure is the same throughout. Figure 2(c) shows a smooth transition of the calculated line shapes upon gradual variation of $\alpha$ from perpendicular to parallel excitation. As the angle $\alpha$ is lowered from $90^{\circ}$ to $0^{\circ}$, the inversion amplitude of the signal increases, the central peak sharpens considerably, and two additional maxima at $B_{0} \approx \pm 0.5 \mathrm{mT}$ develop. For all values of $\alpha$, the predicted signal decays to zero for high strengths of the static magnetic field. The increased noise level and the slight asymmetry in the curves predicted for $\mathbf{B}_{1} \| \mathbf{B}_{0}$ result from the discreteness of the experimental magnetoresistance curve and the resulting error in computing the expected EDMR signal as detailed in Sec. II of the Supplemental Material [25]. Figure 2(d) plots the corresponding experimental EDMR spectra. Remarkably good agreement with the model is found in terms of line shape and amplitude, indicating that at this frequency, the experimental signal is effectively dominated by the quasistatic effect.

In the experiment, additional positive contributions to the signal arise, seen most prominently around $B_{0}=$ $0.5 \mathrm{mT}$ (black arrow), which are not present in the simulation. In Refs. [18,19], very similar deviations between quantum statistical calculations and RYDMR experiments were observed. These deviations presumably originate from the remaining weak Zeeman resonances expected at around $B_{0}= \pm 18 \mu \mathrm{T}$. While one would expect a suppression of the Zeeman resonance for $\mathbf{B}_{1} \| \mathbf{B}_{0}$ in the high-field limit, this is not the case for very weak static external magnetic fields $\mathbf{B}_{0}$ because of the isotropic hyperfine fields. These fields effectively tilt the total static field such that a nonzero component perpendicular to $\mathbf{B}_{1}$ exists $[19,33]$. Careful quantitative analysis of this feature may indeed offer a route to identifying such low-frequency Zeeman resonances by subtraction of the quasistatic contribution, and could be of interest as a laboratory-based probe of models of biological magnetoreception at geomagnetic field strengths [34]. For orientations approaching $\mathbf{B}_{1} \| \mathbf{B}_{0}$, inhomogeneities in the excitation field $\mathbf{B}_{1}$ can cause further slight deviations between experiment and model (see Sec. VI of the Supplemental Material [25] for a discussion of this issue). Another feature that becomes apparent at high excitation powers is an asymmetry between positive and negative $B_{0}$ at a field strength of around $0.5 \mathrm{mT}$, as highlighted by the dashed horizontal line in Fig. 2. This asymmetry is only observed when $\mathbf{B}_{0}$ and $\mathbf{B}_{1}$ approach parallel alignment, which is in agreement with the measurements and calculations performed for circularly polarized excitation in Ref. [19]. The feature coincides with the presence of the additional positive contributions to the signal, 
suggesting a possible link between the two observations. Although this experimental evidence appears to suggest that both effects arise from unresolved Zeeman resonances and imperfect linear polarization of $\mathbf{B}_{1}$, further investigation of both an experimental and theoretical nature is necessary to conclusively determine the possibility of the emergence of resonant contributions for $f \rightarrow 0$.

Obviously, the range of the magnetoresistance curve explored by $\mathbf{B}_{1}$ and thus the magnitude of the quasistatic magnetic field effect changes with the square root of the rf radiation power, $B_{1} \sim \sqrt{P}$. We consider EDMR spectra for different values of the excitation-field strength for perpendicular and parallel excitation conditions. Measurements are conducted for powers ranging from 0.04 to $20 \mathrm{~W}$, from which, for visual clarity, only the results for 0.32 , $1.26,5$, and $20 \mathrm{~W}$ are plotted. The model calculations for these conditions in Figs. 3(a) and 3(c) closely agree with the experimental observations in Figs. 3(b) and 3(d) and notably also yield an rf power dependence of the signal amplitude that matches the experiment. We note that the remaining deviations between calculation and experiment are of the same nature as those discussed for the data in Figs. 2(c) and 2(d). The model calculations show that for both the perpendicular and the parallel excitation configuration, the amplitude of the effect increases with rf field strength in a nonlinear way. This effect is a consequence of the varying curvature of the magnetoresistance profile in the region of the ultrasmall magnetic field effect. The evolution of the line shape exhibits an increasingly pronounced inversion of the signal around $B_{0}= \pm 0.2 \mathrm{mT}$ as $B_{1}$ is increased. This growth of the inversion signal is a consequence of the local functional shape of the magnetoresistance curve around $B_{0}$ in the field region explored by $\mathbf{B}_{1}$. A more detailed discussion of this aspect is provided in Sec. IV of the Supplemental Material [25]. For overly high $\mathbf{B}_{1}$ field strengths, which are presently not accessible experimentally, the model even predicts an entirely inverted signal resembling the static OLED magnetoresistance, as plotted in Figure S3 of the Supplemental Material [25].

\section{Determination of the strength of the rf excitation field $B_{1}$}

The systematic and monotonic variation of EDMR spectral shape with the excitation power allows the determination of $B_{1}$ through comparison of the experimental spectra with model calculations. Only one free parameter is involved in this procedure: the quantity of interest, the value of $B_{1}$ at a given excitation power. For our analysis, we choose the lowest frequency compatible with the experimental equipment, $0.1 \mathrm{MHz}$, to avoid any potential spurious contributions from Zeeman resonances. Experimental spectra recorded with $\mathbf{B}_{1} \perp \mathbf{B}_{0}$ are plotted in Fig. 4 as black lines for two different excitation powers, $1.26 \mathrm{~W}$

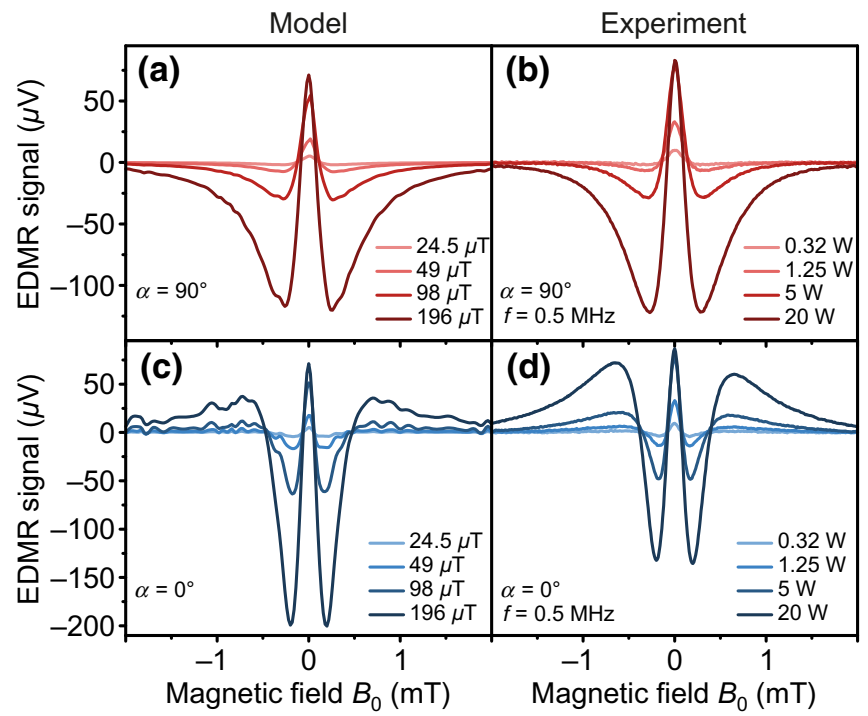

FIG. 3. Power dependence of the quasistatic magnetic field effect. Simulations in panels (a) and (c) are compared to measurements in panels (b) and (d). Red lines (top row) indicate perpendicular orientation of the $\mathbf{B}_{0}$ and $\mathbf{B}_{1}$ fields, blue lines (bottom row) mark parallel alignment.

in panel (a) and $20 \mathrm{~W}$ in panel (b). As the inversion of the line shape depends on the excitation power in a unique manner, the amplitude of $\mathbf{B}_{1}$ can be determined precisely. Excellent agreement between the calculated spectra and the experimentally measured ones is obtained for $B_{1}$ values of 51 and $185 \mu \mathrm{T}$, corresponding to 1.26 and $20 \mathrm{~W}$, respectively. Based on the variation of the line shape with $B_{1}$, these values can be derived with an error of less than $2 \%$. For the lower power, good agreement is found with the simple magnetostatic estimate employed above; for the highest power of $20 \mathrm{~W}$ the magnetostatic estimate gives $195 \mu \mathrm{T}$ compared to the $185 \mu \mathrm{T}$ from the model. The difference in ratios of the two powers and of the squares of the two extracted $\mathbf{B}_{1}$ field strengths (15.87 vs 13.16) suggests a substantial nonlinearity of the dependence of $B_{1}^{2}$ on the nominal rf input power; this effect is most probably caused by heating within the stripline, which results in impedance mismatches in the rf circuit and subsequent power reflection.

To illustrate the capability of this analysis procedure, we perform power-dependent measurements on a second OLED-stripline sample. The nonlinearity is confirmed by measuring the voltage between inner and outer conductor of the rf circuit with an oscilloscope, while exciting the sample at $0.1 \mathrm{MHz}$ and simultaneously acquiring the signal of the quasistatic magnetic field effect as described previously. The excitation power is varied over more than two orders of magnitude from $0.08 \mathrm{~W}$ to $20 \mathrm{~W}$. Plotting the measured squared $\mathrm{rf}$ voltage against the nominal input power reveals the nonlinearity in Fig. 4(c). For each power level, the corresponding value of the excitation field $B_{1}$ 
(a)

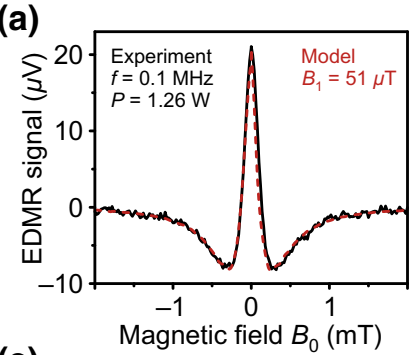

(c)

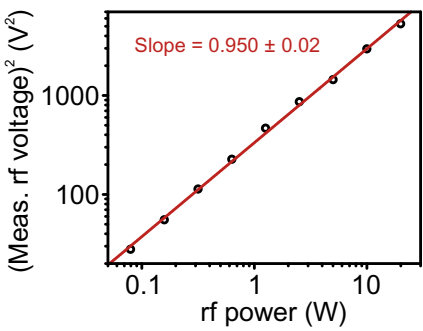

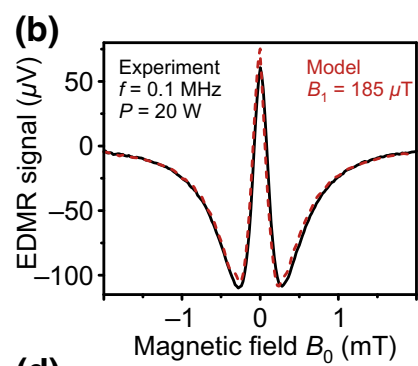

(d)

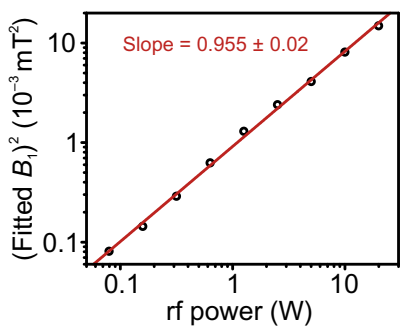

FIG. 4. Determination of the excitation field $B_{1}$ through modeling of the quasistatic field effect. Measured EDMR spectra (black solid lines) taken at $f=0.1 \mathrm{MHz}$ are quantitatively compared with model calculations (red dashed lines) for different $\mathrm{rf}$ excitation powers, $1.26 \mathrm{~W}$ (a) and $20 \mathrm{~W}$ (b). (c) rf voltage, measured between the inner and outer conductor of the rf circuit, for excitation powers from $0.08 \mathrm{~W}$ to $20 \mathrm{~W}$. The squared rf voltage is plotted against the rf power on a double-logarithmic scale, revealing a clear sublinearity. (d) Values for $B_{1}^{2}$ as determined from the fitting procedure in panels (a), (b), plotted as a function of the rf power. The sublinearity observed is, within the fitting error, the same as for the rf voltage.

is determined by the procedure described in Figs. 4(a) and 4(b). As before, very good agreement between the experimental signal and the calculated spectrum arising from the quasistatic field effect is found. The results are summarized in Fig. 4(d), where $B_{1}^{2}$ is plotted as a function of the rf input power. Comparing the plots of the power dependence of the measured voltage drop [panel (c)] and the inferred rf magnetic field strength [panel (d)] confirms that the nonlinearity detected in the voltage of the rf circuit is indeed reproduced with high fidelity by the determination of the strength of $\mathbf{B}_{1}$ using the line-shape analysis described previously.

Satisfactory agreement between experiment and model calculations is also found for the case of $\mathbf{B}_{1} \| \mathbf{B}_{0}$, marred only slightly by the aforementioned asymmetry and the additional positive signal contributions in the low-field EDMR spectra that are not reproduced by the model (see Figure S4 in Sec. V of the Supplemental Material [25]). This agreement offers a further indication of the consistency of our model and analysis.

The method presented in this section constitutes a procedure to determine the excitation-field strength $B_{1}$ in the absence of Zeeman resonances, i.e., when an analysis of power broadening or a direct measurement of the Rabi

frequency is not possible. The method is applicable over a wide range of excitation-field strengths, presently limited only by the experimental equipment. Assuming that the power-to-field conversion factor is not strongly dependent on the excitation frequency, the results obtained for low frequencies, where the spectrum is dominated by the quasistatic field effect, can be extrapolated to higher $f$, where Zeeman resonances also emerge. This potential for extrapolation is corroborated by the fact that the $B_{1}$ values determined here show remarkably good agreement with previous estimates based on power broadening of the Zeeman resonance at a much higher frequency of $f=280 \mathrm{MHz}$ [12], and the corresponding magnetostatic calculations based on the stripline geometry.

\section{SUMMARY AND OUTLOOK}

We present EDMR measurements of OLEDs in the ultralow-frequency regime, where the spin- $1 / 2$ Zeeman resonances appear to become suppressed and qualitatively new spectral features emerge, originating from a quasistatic magnetic field effect. Comparison between a simple model, based on an intuitive vector addition of the static and oscillating magnetic fields, and measurements over a wide range of experimental parameters allows us to precisely determine the local $\mathbf{B}_{1}$ field amplitude of the $\mathrm{rf}$ radiation. We stress that this procedure does not rely on any assumptions regarding the microscopic material properties, such as the strength of spin-spin interactions or the charge-carrier pair recombination and dissociation rates. The effects of these additional variables are implicitly contained in the static magnetoresistance behavior of the OLED, which is then used to predict the line shape of the quasistatic effect in the EDMR spectra.

There have been extensive efforts to develop models accounting for the magnetoresistance behavior of OLEDs and organic semiconductors in general. A theory aiming to correctly describe the static magnetic field effects of OLEDs should also reproduce the characteristics of the quasistatic magnetic field effect in EDMR accurately, as this effect is a direct consequence of the magnetoresistance behavior. Since the quasistatic effect is sensitive to the derivatives of the magnetoresistance profile, we anticipate that this effect will serve as fertile ground for testing model assumptions and constraining microscopic parameters of the carrier-pair model of magnetoresistance in organic semiconductors [35-37].

There is still a wealth of information to be gained from a detailed analysis of the evolution from EDMR spectra dominated by Zeeman resonances to a frequency regime where the signal arises exclusively due to the quasistatic effect. The decomposition of the EDMR spectrum into Zeeman and quasistatic contributions could potentially yield information on the functional shape of the 
distribution of carrier-pair coherence times. Even though the average of the coherence times as determined by spinecho spectroscopy is only of order $300 \mathrm{~ns}[22,29]$, there may conceivably be a small subset of pairs with much longer coherence times that are not visible in the conventional echo experiment. A successful model of low-field EDMR should correctly account for the contribution of carrier pairs with coherence times of the order of the rf period. This will provide information on the crucial parameter of the carrier-pair model, the pair coherence time, by exploiting two phenomena, a resonant and a nonresonant one. Finally, we stress that this ultralow field regime is particularly interesting for probing the exotic nonperturbative regimes of ultrastrong or even deep-strong drive, where the Rabi frequency of the spin system exceeds its Larmor frequency. Interesting physics, such as the spinDicke effect and photon-dressed spin states, emerge in this regime of resonantly driven spin transitions [33,3840]. With a quantitative understanding of EDMR spectra at ultralow frequencies, we expect to be able to identify signatures of deep-strong driving, which would otherwise require unphysically large $B_{1}$ field strengths [33].

\section{ACKNOWLEDGMENTS}

The authors would like to thank Dr. Hans Malissa for fruitful discussions and Sebastian Milster for preliminary experiments. This work is funded by the Deutsche Forschungsgemeinschaft (DFG, German Research Foundation) (Project ID 314695032 - SFB 1277). P.L.B. acknowledges support as an Australian Research Council Laureate Fellow (FL160100067). The National Deuteration Facility is partly funded by NCRIS, an Australian Government initiative.

[1] D. R. McCamey, H. A. Seipel, S.-Y. Paik, M. J. Walter, N. J. Borys, J. M. Lupton, and C. Boehme, Spin Rabi flopping in the photocurrent of a polymer light-emitting diode, Nat. Mater. 7, 723 (2008).

[2] K. J. van Schooten, D. L. Baird, M. E. Limes, J. M. Lupton, and C. Boehme, Probing long-range carrier-pair spin-spin interactions in a conjugated polymer by detuning of electrically detected spin beating, Nat. Commun. 6, 6688 (2015).

[3] S. Milster, T. Grünbaum, S. Bange, S. Kurrmann, H. Kraus, D. M. Stoltzfus, A. E. Leung, T. A. Darwish, P. L. Burn, C. Boehme, and J. M. Lupton, Perdeuterated conjugated polymers for ultralow-frequency magnetic resonance of OLEDs, Angew. Chemie - Int. Ed. 59, 9388 (2020).

[4] E. W. Knapp and K. Schulten, Magnetic field effect on the hyperfine-induced electron spin motion in radicals undergoing diamagnetic-paramagnetic exchange, J. Chem. Phys. 71, 1878 (1979).
[5] V. N. Prigodin, J. D. Bergeson, D. M. Lincoln, and A. J. Epstein, Anomalous room temperature magnetoresistance in organic semiconductors, Synth. Met. 156, 757 (2006).

[6] S. P. Kersten, A. J. Schellekens, B. Koopmans, and P. A. Bobbert, Magnetic-Field Dependence of the Electroluminescence of Organic Light-Emitting Diodes: A Competition Between Exciton Formation and Spin Mixing, Phys. Rev. Lett. 106, 197402 (2011).

[7] U. E. Steiner and T. Ulrich, Magnetic field effects in chemical kinetics and related phenomena, Chem. Rev. 89, 51 (1989).

[8] B. Brocklehurst, Magnetic fields and radical reactions: recent developments and their role in nature, Chem. Soc. Rev. 31, 301 (2002).

[9] K. Maeda, K. B. Henbest, F. Cintolesi, I. Kuprov, C. T. Rodgers, P. A. Liddell, D. Gust, C. R. Timmel, and P. J. Hore, Chemical compass model of avian magnetoreception, Nature 453, 387 (2008).

[10] V. Dyakonov, G. Rösler, M. Schwoerer, and E. L. Frankevich, Evidence for triplet interchain polaron pairs and their transformations in polyphenylenevinylene, Phys. Rev. B 56, 3852 (1997).

[11] H. Malissa, R. Miller, D. L. Baird, S. Jamali, G. Joshi, M. Bursch, S. Grimme, J. van Tol, J. M. Lupton, and C. Boehme, Revealing weak spin-orbit coupling effects on charge carriers in a $\pi$-conjugated polymer, Phys. Rev. B 97, 161201 (2018).

[12] T. Grünbaum, S. Milster, H. Kraus, W. Ratzke, S. Kurrmann, V. Zeller, S. Bange, C. Boehme, and J. M. Lupton, OLEDs as models for bird magnetoception: detecting electron spin resonance in geomagnetic fields, Faraday Discuss. 221, 92 (2020).

[13] T. D. Nguyen, B. R. Gautam, E. Ehrenfreund, and Z. V. Vardeny, Magnetoconductance Response in Unipolar and Bipolar Organic Diodes at Ultrasmall Fields, Phys. Rev. Lett. 105, 166804 (2010).

[14] C. R. Timmel and P. J. Hore, Oscillating magnetic field effects on the yields of radical pair reactions, Chem. Phys. Lett. 257, 401 (1996).

[15] J. R. Woodward, R. J. Jackson, C. R. Timmel, P. J. Hore, and K. A. McLauchlan, Resonant radiofrequency magnetic field effects on a chemical reaction, Chem. Phys. Lett. 272, 376 (1997).

[16] J. R. Woodward, C. R. Timmel, K. A. McLauchlan, and P. J. Hore, Radio Frequency Magnetic Field Effects on Electron-Hole Recombination, Phys. Rev. Lett. 87, 077602 (2001).

[17] K. B. Henbest, P. Kukura, C. T. Rodgers, P. J. Hore, and C. R. Timmel, Radio frequency magnetic field effects on a radical recombination reaction: A diagnostic test for the radical pair mechanism, J. Am. Chem. Soc. 126, 8102 (2004).

[18] C. T. Rodgers, K. B. Henbest, P. Kukura, C. R. Timmel, and P. J. Hore, Low-Field optically detected EPR spectroscopy of transient photoinduced radical pairs, J. Phys. Chem. A 109, 5035 (2005).

[19] C. J. Wedge, C. T. Rodgers, S. A. Norman, N. Baker, K. Maeda, K. B. Henbest, C. R. Timmel, and P. J. Hore, Radiofrequency polarization effects in low-field electron paramagnetic resonance, Phys. Chem. Chem. Phys. 11, 6573 (2009). 
[20] S. Jamali, G. Joshi, H. Malissa, J. M. Lupton, and C. Boehme, Monolithic OLED-microwire devices for ultrastrong magnetic resonant excitation, Nano Lett. 17, 4648 (2017).

[21] W. J. Baker, D. R. McCamey, K. J. van Schooten, J. M. Lupton, and C. Boehme, Differentiation between polaron-pair and triplet-exciton polaron spin-dependent mechanisms in organic light-emitting diodes by coherent spin beating, Phys. Rev. B 84, 165205 (2011).

[22] D. M. Stoltzfus, G. Joshi, H. Popli, S. Jamali, M. Kavand, S. Milster, T. Grünbaum, S. Bange, A. Nahlawi, M. Y. Teferi, S. I. Atwood, A. E. Leung, T. A. Darwish, H. Malissa, P. L. Burn, J. M. Lupton, and C. Boehme, Perdeuteration of poly[2-methoxy-5-(2'-ethylhexyloxy)1,4-phenylenevinylene] (d-MEH-PPV): control of microscopic charge-carrier spin-spin coupling and of magnetic-field effects in optoelectronic devices, J. Mater. Chem. C 8, 2764 (2020).

[23] M. Kavand, D. Baird, K. van Schooten, H. Malissa, J. M. Lupton, and C. Boehme, Discrimination between spin-dependent charge transport and spin-dependent recombination in $\pi$-conjugated polymers by correlated current and electroluminescence-detected magnetic resonance, Phys. Rev. B 94, 075209 (2016).

[24] W. J. Baker, K. Ambal, D. P. Waters, R. Baarda, H. Morishita, K. van Schooten, D. R. McCamey, J. M. Lupton, and C. Boehme, Robust absolute magnetometry with organic thin-film devices, Nat. Commun. 3, 898 (2012).

[25] See Supplemental Material at http://link.aps.org/supple mental/10.1103/PhysRevApplied.15.064001 for details on the magnetostatic estimation of the excitation field $B_{1}$ and on the numerical procedure for computing the quasistatic magnetic field effect including model calculations for large excitation-field strengths. The Supplemental Material includes Refs. [26,27].

[26] Ö Mermer, G. Veeraraghavan, T. L. Francis, and M. Wohlgenannt, Large magnetoresistance at roomtemperature in small-molecular-weight organic semiconductor sandwich devices, Sol. Stat. Commun. 134, 631 (2005).

[27] W. Ratzke, S. Bange, and J. M. Lupton, Direct Detection of Singlet-Triplet Interconversion in OLED Magnetoelectroluminescence with a Metal-Free FluorescencePhosphorescence Dual Emitter, Phys. Rev. Appl. 9, 054038 (2018).

[28] J. A. Weil and J. R. Bolton, Electron Paramagnetic Resonance, 2nd ed (Wiley, New Jersey, 2007).
[29] W. J. Baker, T. L. Keevers, J. M. Lupton, D. R. McCamey, and C. Boehme, Slow Hopping and Spin Dephasing of Coulombically Bound Polaron Pairs in an Organic Semiconductor at Room Temperature, Phys. Rev. Lett. 108, 267601 (2012).

[30] J. Kalinowski, J. Szmytkowski, and W. Stampor, Magnetic hyperfine modulation of charge photogeneration in solid films of $\mathrm{Alq}_{3}$, Chem. Phys. Lett. 378, 380 (2003).

[31] C. Kerpal, S. Richert, J. G. Storey, S. Pillai, P. A. Liddell, D. Gust, S. R. Mackenzie, P. J. Hore, and C. R. Timmel, Chemical compass behaviour at microtesla magnetic fields strengthens the radical pair hypothesis of avian magnetoreception, Nat. Commun. 10, 3707 (2019).

[32] P. Klemm, S. Bange, A. Pöllmann, C. Boehme, and J. M. Lupton, Nanotesla magnetoresistance in $\pi$-conjugated polymer devices, Phys. Rev. B 95, 241407 (2017).

[33] S. Jamali, V. V. Mkhitaryan, H. Malissa, A. Nahlawi, H. Popli, T. Grünbaum, S. Bange, S. Milster, D. Stoltzfus, A. E. Leung, T. A. Darwish, P. L. Burn, J. M. Lupton, and C. Boehme, Floquet spin states in OLEDs, Nat. Commun. 12, 465 (2021).

[34] T. Ritz, P. Thalau, J. B. Phillips, R. Wiltschko, and W. Wiltschko, Resonance effects indicate a radical-pair mechanism for avian magnetic compass, Nature 429, 177 (2004).

[35] D. R. McCamey, S.-Y. Lee, S.-Y. Paik, J. M. Lupton, and C. Boehme, Spin-Dependent dynamics of polaron pairs in organic semiconductors, Phys. Rev. B 82, 125206 (2010).

[36] A. J. Schellekens, W. Wagemans, S. P. Kersten, P. A. Bobbert, and B. Koopmans, Microscopic modeling of magnetic-field effects on charge transport in organic semiconductors, Phys. Rev. B 84, 075204 (2011).

[37] S. L. Bayliss, N. C. Greenham, R. H. Friend, H. Bouchiat, and A. D. Chepelianskii, Spin-Dependent recombination probed through the dielectric polarizability, Nat. Commun. 6, 8534 (2015)

[38] R. C. Roundy and M. E. Raikh, Organic magnetoresistance under resonant ac drive, Phys. Rev. B 88, 125206 (2013).

[39] D. P. Waters, G. Joshi, M. Kavand, M. E. Limes, H. Malissa, P. L. Burn, J. M. Lupton, and C. Boehme, The spin-Dicke effect in OLED magnetoresistance, Nat. Phys. 11, 910 (2015).

[40] V. V. Mkhitaryan, D. Danilović, C. Hippola, M. E. Raikh, and J. Shinar, Comparative analysis of magnetic resonance in the polaron pair recombination and the triplet excitonpolaron quenching models, Phys. Rev. B 97, 035402 (2018). 